\title{
Between Pricing and Investment, What Mobility Policies Would Be Advantageous for Île-de-France?
}

\author{
Fabien Leurent $(\mathbb{D}$ ) and Sheng Li \\ Ecole des Ponts ParisTech, LVMT, Marne la Vallée, France \\ Correspondence should be addressed to Fabien Leurent; fabien.leurent@enpc.fr
}

Received 22 May 2020; Revised 17 November 2020; Accepted 4 December 2020; Published 21 December 2020

Academic Editor: Jose E. Naranjo

Copyright (C) 2020 Fabien Leurent and Sheng Li. This is an open access article distributed under the Creative Commons Attribution License, which permits unrestricted use, distribution, and reproduction in any medium, provided the original work is properly cited.

\begin{abstract}
This article provides a prospective study of mobility policies for the private car and public transit (PT) modes of transportation in the Paris Ile-de-France region. Different economic instruments are considered: pricing of car traffic or transit service, subsidizing PT, and investment in PT to improve service quality. Policy scenarios are defined and assessed according to multiple criteria: users' benefits, PT production costs and fare revenues, public subsidies, and environmental damage both local (air pollution) and global (carbon emissions). The social, economic, and environmental impacts are monetized and aggregated in a wellbeing function. While a first set of scenarios are specified directly, two other sets of scenarios are calculated by optimizing the wellbeing function with respect to action variables on the transit mode in the medium or long run. The regional mobility system is modeled in a structural way: concentric subregions, travel demand segmented by geographical and behavioral conditions, environmental impacts based on road and rail traffic, and car mode and transit mode depicted each as a set of technical components involving 1 to 3 structural factors that can make action levers. This model-based methodology allows for trading between different kinds of impacts and identifying performance-oriented policy packages.
\end{abstract}

\section{Introduction}

In an urban territory, the mobility system provides access to the places of activity and accommodation. People can get from place to place by using travel modes. For medium to long distance trips, motorized modes such as the private car (PC) and public transit (PT) by bus or train are convenient to their users. Yet, the motorized modes damage the environment, not only by the consumption of matters and energy, but also by the emission of greenhouse gases, air pollutants, and noise. The last two items are of special concern to the dwellers living in the vicinity of roadway traffic.

To foster sustainable development, urban mobility plans have been oriented to the development of noncar modes, including the transit network together with walking and bicycles. The so-called Sustainable Urban Mobility Plans (SUMPs) are designed for the short to medium terms [1].
The development of transit modes is faced to the limited availability of fare revenues and of public funds.

In this article, we take a long-term perspective to explore the potential development of transit modes in a metropolitan territory: that of Greater Paris region called "Ile-de-France." We look for structural recomposition of the transit network in terms of submodes (bus, metro, and train) in subregions (central area, inner ring, and outer ring), so as to maximize the collective benefits to users, operators, and the environment. Both the transit mode and the car mode are considered, with special emphasis on mode choice on the basis of service quality, travel time, and money costs which involve transit fares and optionally road charges. The costs of transit implementation and operation are considered, too, in relation to transit revenues and the amount of public subsidies. Two cases of public funding are addressed, with fixed or unlimited amount of subsidies: the latter case will enable us to assess which level of public 
funding would be optimal for the sustainable development of the territorial mobility system.

Our study methodology involves a strategic model of mobility demand and transportation supply: we adapt the STEM model [2] to deal with origin-destination transit fares and variable public funding. The model enables for synthetic representation of the modal networks on the basis of a restricted set of structural parameters, which constitute action levers for mobility policy making. The model will be used in two ways: either to assess specific plans with preset values of the structural factors or to look for an optimal plan by maximizing an objective function, optionally subjected to a budget constraint. In the latter way, the structural factors are endogenous to the model. By specifying which subset is endogenous among the set of structural factors, we shall specify medium-term scenarios that involve transit fares and frequencies and long-term scenarios that add line lengths and station densities.

The rest of the article is organized in five sections. After reviewing related work (Section 2), we provide an overview of the strategic model (Section 3). We then introduce the territory and mobility system under study (Section 4), before exploring a range of policy scenarios (Section 5). To conclude, we shall discuss the outreach and limitations of the study and point to directions of further research (Section 6).

\section{Related Work}

Related work about network development and service pricing can be divided in three streams. On the one hand, there are essentially theoretical contributions by transportation economists for abstract situations (Section 2.1). On the other hand, there are operations research models that detail space and traffic conditions (Section 2.2). Between these two streams, a sort of hybrid stream has gradually emerged, which synthesizes the spatial detail to deal with planning policy at a high level (Section 2.3).

2.1. Theoretical Principles and Abstract Models. Limited public funds constrain the choices between different investment projects and between different mobility policies. In order to support decision-making in this domain, transport economists have long sought to establish theoretical principles, rules, and models. As far back as 1844, Jules Dupuit modelled the surplus that users would obtain from a new road, to balance it against the costs of production and revenues from pricing [3]. Pigou [4] studied the distribution of traffic between two competing routes: he modelled the marginal social cost (MSC) of each route, incorporating the phenomenon of congestion, and showed that a price based on marginal cost would steer the traffic in a way that is optimal for the system. Wardrop [5] extended Pigou's analysis by considering several alternative routes on a road network. Walters [6] added to the marginal social cost a valuation of environmental impacts. Vickrey [7] considered more options for individual mobility: not only cars but also transit and, for each mode the possibility of choosing the departure time in order to have access to a variety of travel conditions. Mohring [8] considered an idealised transit line, consisting of stations that are homogenous in terms of spacing and demand volumes, in order to optimise the local authority's trade-offs between the spacing of the stations and the size of the fleet, on the basis of their respective effects on the service provided to users and on the costs of production. He also set an optimum price level for the line as a transit subsystem.

2.2. Spatially Detailed Models for Network Planning. Back in 1955, Martin Beckmann [9] pioneered a new research stream, looking at a whole road network and a variety of car paths between a set of origins and destinations. Beckmann modelled Wardrop's two principles: the user equilibrium and the system optimum, in the form of two typical problems of operational research combining graph theory and optimisation theory. The framework of the model was adopted, adapted, and extended in numerous directions, for example, by Spiess and Florian [10] and Cepeda et al. [11] for transit, Li et al. [12] for static multimodal assignment, Kuwahara and Akamatsu [13] for dynamic roadway traffic assignment, and Meschini et al. [14] for dynamic multimodal assignment.

The principle of treating a network at the detailed level of arcs and nodes became dominant in traffic studies intended to support decision-making in transport planning [15]. It has been adapted for use in structural network planning: among many others, Leblanc [16] and Cantarella and Vitetta [17] modelled the development of flow capacities on road links on the basis of flow volumes and costs, whereas Lee and Vuchic [18] modelled the structuring of a PT network in terms of routes and vehicle fleets and so did Murray [19] in order to optimise the coverage of a territory's mobility needs.

\subsection{A Hybrid Research Current, Strategic Modelling of Urban} Systems. Between the two abovementioned research streams, a sort of hybrid has gradually emerged which considers both the spatial structure of transport supply and mobility demand and the economic structure of transport modes in terms of infrastructure, vehicle fleet, service conditions, and production costs. This hybrid category consists of "strategic" models of transport in a conurbation. The purpose of the strategic model is to represent a concrete situation in synthetic form, in order to elucidate the political planning and management strategy across a whole agglomeration.

The thesis by Van Nes [20] is a seminal work that picks up, systematizes, and develops strategic models for each transport mode and also for several transport modes, along hierarchical lines. In particular, Van Nes succeeded in describing transport networks with small sets of structural parameters: the length of lines, the spacing of stations, and the size of the vehicle fleet, in the case of a transit system. In this way, he was able to model the optimum design of a network as a problem of continuous optimisation with respect to such structural parameters, linked by constraints of a technical nature. Combes and Van Nes [21] proposed a hierarchical representation for a transit network consisting 
of both road and rail lines, as well as spatialisation by concentric subregions for a monocentric agglomeration: they applied their model to the case of the Paris agglomeration. Complementarily, Leurent et al. [22] provided a mathematical formulation and a theoretical analysis to characterize the optimum constitution of a transit network in the economic form of four "golden rules" linking, respectively, length of lines, spacing between stations, fleet size, and pricing, to macroscopic indicators of transport supply and demand.

By modelling an urban network of bus routes as a regular grid, Daganzo [23] addressed the problem of optimum design in a completely analytical way. This analytical geometry of transport networks was applied by Estrada et al. [24] to a rectangular PT network with a set of structural parameters for each of the two axes and then by Badia et al. $[25,26]$ for different city and network shapes. In a similar vein, Zhang et al. [27] addressed trunk-and-branches networks to design bus services in an optimal way, comparing semiautonomous and fully autonomous driving technologies.

Daganzo and Geroliminis [28] proposed modelling a road network and road traffic conditions by means of a macroscopic fundamental diagram (MFD), a function that links average traffic speed with structural network parameters and with an indicator for aggregated flow volume. There have been multiple contributions to MFD theory, both empirical $[29,30]$ and theoretical $[31,32]$. Among the economic developments, Zheng et al. [33] modelled timetable choice and dynamic use pricing on the road network, and Wei and Sun [34] also addressed the issue of route choice on a heterogeneous network with two types of roads.

In order to include both automobile and transit modes, modal share and environmental impacts, Estrada et al. [35] modelled the road network with an MFD, the bus lines with a form of two-dimensional grid, demand as a set of trips with different origin-destination relations, for each trip with the modal choice between the private car and transit on the basis of a discrete choice logit model, and the environmental impacts of road vehicle traffic. They numerically minimised a collective cost function that included user surplus (with fixed volume of demand), the production costs of transit, and the monetisation of energy consumption and pollutant emissions. The model developed by Leurent et al. [2] includes an MFD model of the road network for each subregion, a hierarchical transit model (distinguishing between the bus, metro, and train submodes), and a more closegrained breakdown of the environmental impacts, giving spatial details of the levels of exposure to atmospheric pollution. Moreover, users are modelled in terms of their multimodal flexibility or conversely their dependency on the car or transit mode.

\section{Overview of the STEM Model}

Figure 1 introduces the architecture of the STEM model. It combines submodels of (i) travel demand and mode choice, (ii) car mode, (ii) transit mode, (iv) environmental impacts, and (v) policy composition. Before giving a short description of each submodel, let us first state the modeling scales according to space and time.

Space is represented in two respects: subregions to locate places in the territory and demand segments for trip-making situations. The territory, by assumption an urban region, is divided in several subregions: central area and concentric rings for suburbs from "inner" to "outer." By subregion, each transportation mode is modelled as a "component" according to a small set of structural parameters also called factors, which summarize statistically the subnetwork composition and enable one to derive its quality of service, trip time, and price conditions. The trip-making situations are segmented with respect to the subregions of origin and destination but also to other conditions, including distance subrange.

As for the time dimension, two scales are of interest: the day and the year. Network conditions and operations are described per average working day, as well as the trips, the generalized costs of modal options to their users, the mode frequentation, and the environmental impacts. Conversion from day to year of the physical quantities involves a year equivalent number of working days, denoted by Y. The economic aggregates are evaluated on a yearly basis. "Annual" production costs include operational costs and maintenance costs and also investment costs taken in a lifecycle perspective.

3.1. Mobility Demand. Mobility demand is modelled as a set of trips segmented according to geographical and behavioural types. The geographical types pertain to the origin and destination pair of subregions and to the distance subrange (short, medium, or long) and possibly also to a routing option (via the central area or bypassing it). The behavioural type depicts the user's situation with respect to the two motorized modes: either car-dependent, or transit dependent, or mode flexible (chooser).

Per trip and per modal option for it, the quality of service (travel time) and the money cost to its user are modelled. The trip "generalized cost" to its user adds up the trip money cost and the travel time multiplied by a money "value of time."

For those users having mode flexibility, the modal split is modelled on the basis of the respective generalized costs of the two modes, according to a binary logit discrete choice model.

3.2. Car Mode and Road Network. The car mode combines the private car of the user with the roadway infrastructure. The physical and economic conditions of the trip path depend on the vehicle type and its "impact factors" by kind of impact (e.g., energy consumption) and per unit of distance. Vehicle type can be described as a behavioural dimension of demand segmentation, or the different types can be aggregated into a weighted average, thus constituting a "composite car."

The car trip generates money costs: energy costs depending on the path composition (distance travelled in each subregion) and traffic conditions, destination parking price, and, in some circumstances, road tolls. All of these are 


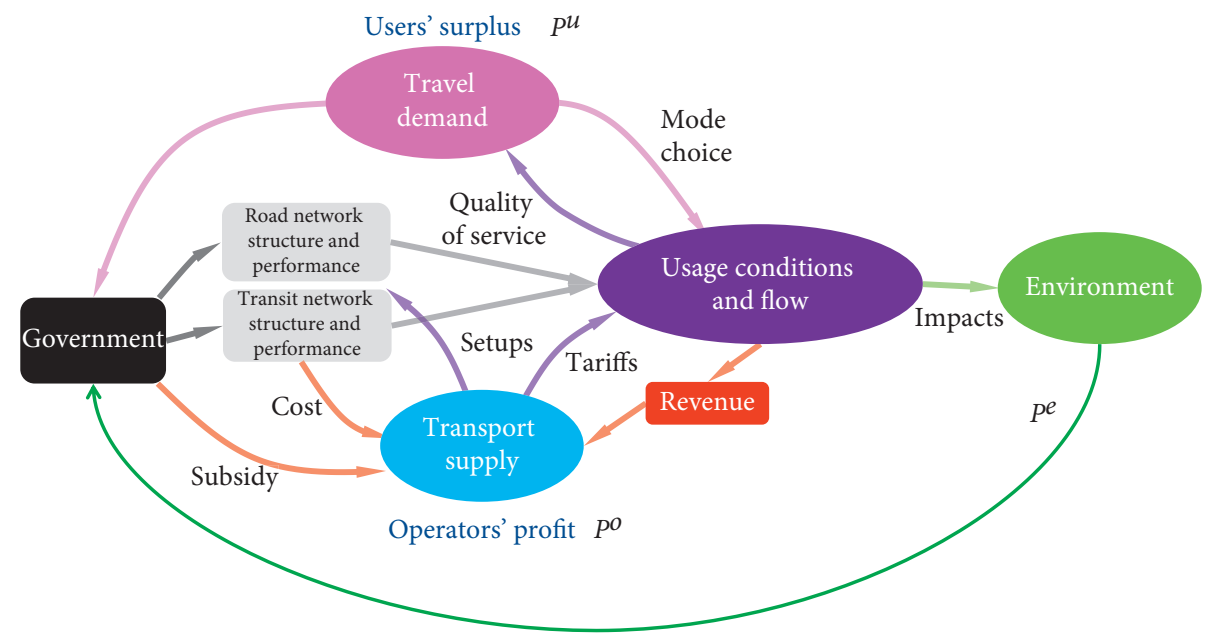

FIGURE 1: Diagram of the system as modelled.

added up to the ownership cost so as to constitute the full money cost of the car trip. Path usage will also take time to its user, depending on the travelled distances and the running speed. The trip time is multiplied by a "value of time" to give the trip time cost, which is added up to the money cost in order to constitute the generalized cost of the car tip to its user.

3.2.1. Roadway Traffic and Network. The users' car trips along the roadway network generate traffic quantities in veh.km (travelled distances). One roadway subnetwork is modelled by subregion: the total traffic quantity is faced to a "flowing capacity" measured in an equivalent mileage of monodirectional lanes that would flow without interruption. The ratio between the demanded traffic and the flowing capacity determines the average speed of the roadway component, through a mathematical function called "macroscopic fundamental diagram" [28].

3.3. Transit Modes. A transit mode combines infrastructures and vehicles of a given kind to offer "trip legs" from station to station along lines. To the user, every transit trip consists in one or several in-vehicle legs, each with its pedestrian phases of access, waiting, and egress or transfer. In the STEM model, the transit network is represented as a set of "components," each of which corresponds to a subregion and a submode (e.g., bus, metro, and train). A component is described by three structural factors: the line length $L_{r}$, the number of stations $\sigma_{r}$, and the quantity of rolling stock $N_{r}$. Based on these, four technical relationships derive specific outcomes of, respectively, (i) station spacing, (ii) commercial speed, (iii) service frequency, and (iv) pedestrian distance of station access at the trip origin (or destination).

In turn, the resulting characteristics induce elemental travel times per trip leg and by phase of access, waiting, invehicle riding, and egress. From the phase times in the different components, by linear combination with weights that depend on the origin-destination pair and demand segment, stems the trip travel time. The weights are similar to the hyperpath-link coefficients in typical models of traffic assignment to a transit network [36]. They are obtained by statistical treatment of a Household Travel Survey.

Like for the car mode, the generalized cost of a trip using the transit mode is defined as the sum of the fare and the travel times weighted by money value-of-times that depend on the leg phases.

3.3.1. Production Means and Costs. On the supply side, the production costs are modelled for each component on the basis of its structural factors $L_{r}, \sigma_{r}$, and $N_{r}$, with respective unit costs of $c_{r}^{L}, c_{r}^{\sigma}$, and $c_{r}^{N}$ which include the operational cost and the capital costs spread over the lifetime span. By linear combination of the structural factors weighted by their respective unit costs, the operation cost of the component to the transit operator is obtained. To this are added "commercial costs" proportional to the number of trips made using the component.

Reciprocally, the transit trips give rise to fare revenues: a specific transit fare is modeled by demand segment according to its geographical conditions (origin-destination pair, etc.).

3.4. Environmental Impacts. The impacts of a transportation system on the environment are multiple and occur at both construction and usage phases in the lifecycles of its infrastructures, vehicles, and consumables. There are local impacts such as noise and air pollution, as well as global impacts such as carbon emissions. The level of the local impacts depends not only on the quantity emitted but also on the population exposed to it locally: hence, local population density must be included in the economic valuation [37].

Here, we consider two types of impacts, namely, (i) air and noise pollution from private cars and also from transit vehicles either buses or trains and (ii) greenhouse gas emissions measured in carbon equivalent. Both types of emissions depend on vehicle traffic (travelled distances by 
kind of vehicle), motor type and power, and average running speed.

3.5. Economic Indicators and Wellbeing Function. Mobility stakeholders in the territory include the users, the operators, and also the environment in which residents are exposed to local impacts. The users' surplus consists of the surplus of mode-choosers (log-sum formula for a logit discrete choice model) and of dependent users (generalized costs).

On a per year basis, one indicator of profit is evaluated for each kind of stakeholders in the modelled mobility system: let us denote by $P_{Y}^{u}$ the users' surplus, $P_{Y}^{o}$ the profit function of the transit operators, and $P_{Y}^{e}$ the environmental benefits (the opposite of the environmental cost function that sums up the different kinds of impact quantities, each multiplied by a specific unit shadow price). From the categorical profit functions stem the collective "wellbeing functions," either $W_{1}=P_{Y}^{u}+P_{Y}^{o}$ neglecting the environment or $W_{0}=P_{Y}^{u}+P_{Y}^{o}+P_{Y}^{e}$ including the environment.

3.6. Policy Design as a Mathematical Program. The wellbeing function depends on a large and varied set of parameters: energy prices, modal tariffs, the structural factors of transit components, and the average speeds of roadway components. The level of public subsidies to transit supply, denoted by $S_{Y}$, can be assumed exogenous or endogenous.

The policy design problem is modeled as a mathematical program for optimizing overall wellbeing, by acting on the decision variables, and subject to the requirement of transit supply profitability as well as technical constraints (quality of service, elemental times, generalized costs of the modes, and traffic operational processes); cf. Leurent et al. [38].

We implemented the model in Python language and used the Optimization algorithm from the SciPy library in order to compute an optimal state. In our application to the case of the Paris agglomeration, the action vector includes 57 scalar variables: there are 3 subareas, 21 demand segments, and 11 transit components and hence 21 transit fares plus $11 \times 3$ structural parameters for the transit mode and 3 zone average speeds for the car mode.

\section{Reference Situation: Territorial and Macroeconomic Analysis}

4.1. Territory and Travel Demand. As of 2010, Greater Paris had a population of 11.6 million in an urbanised area of $1250 \mathrm{~km}^{2}$ within the Île-de-France region, an area of 12 $100 \mathrm{~km}^{2}$. The regional area is split into three subareas, namely, the city of Paris, the inner suburbs, and outer suburbs (see Figure 2(a)).

Our synthetic description of motorized transport networks is based on the MODUS transport demand model by DRIEA (the National Regional Planning Agency). It is a four-step model, from which we drew the respective lane lengths of the subregional road networks, as well as the statistical indicators of the public transport networks (Figures 2(b) and 2(c)). The public transport system consists of four submodes $(m)$ : the RER mode is a set of train lines connecting peripheries by crossing the centre of the urban area (the green line in Figure 2(d)), the Transilien mode is a set of radial railway lines drawing on the periphery from a hub in the centre (the purple line in Figure 2(d)), there is the metro whose lines irrigate the central part and its immediate surroundings (the red line in Figure 2(e)), and we have the bus lines (Figure 2(f)).

According to the regional Household Travel Survey "EGT 2010," motorized travel demand consists of 15.5 million car trips and 8.3 million public transport trips, on average per working day. These trips can be divided into three types: 7.9 million car-dependent trips, a further 8.4 million trips that are made by car but flexible, plus 8 million PT trips, of which 3 million are flexible and 5 million are dependent. On average, PT trips are slightly longer in distance and markedly longer in time than their counterparts by private car (Table 1). The trips are also disaggregated in 21 geographical segments, by O-D pair of subregions, straight line distance subrange (below $3 \mathrm{~km}, 3$ to $10 \mathrm{~km}$, and above $10 \mathrm{~km}$ ), and the binary alternative via either the central area or bypassing it. For flexible users, the binary logit model of modal choice was estimated with a coefficient of $\theta=.27$ and a modal malus of $\zeta=€ 3$ for PT.

4.2. Car Mode and Road Network. The average car model was defined by combination of diesel and gasoline fleets, yielding average price of $€ 1.2$ per litre of fuel. The energy consumption function according to speed is taken from Copert [39]. A money cost per unit distance of $€ .4 / \mathrm{km}$ encompasses energy costs as well as car ownership costs. A multiplying factor of 1.4 is applied to straight-line distances in order to obtain network distances. Average occupancy rate is 1.2 persons per car. Table 1 presents the major indicators of the three roadway components.

4.3. Transit Mode. By coupling the three subareas and the four transit submodes, 11 transit components were obtained. Table 2 shows the components and indicates the synthetic descriptors, together with usage statistics [40]. An average price of $€ 1.0$ per trip was estimated on the basis of the 2010 Household Travel Survey and commercial statistics of the regional transit system.

As concerns transit production costs, we considered the operating costs for a normal year, including operations and maintenance but excluding investment for the development of new lines. The costs are declared by the operators for each transport mode and each function, distinguishing between general functions (management, marketing, research, etc.) and specific functions (energy provision, operations, and maintenance), each according to technical subsystems of lines, stations, and rolling stock. We related the specific costs to the synthetic descriptors $L_{r}, \sigma_{r}$, and $N_{r}$ to derive their respective unit costs on an annual basis. Then, we divided the costs of the general functions by the number of days and daily passenger trip volume, to obtain a unit "commercial cost" of $c_{1}^{t}=€ 0.8$ per trip. 


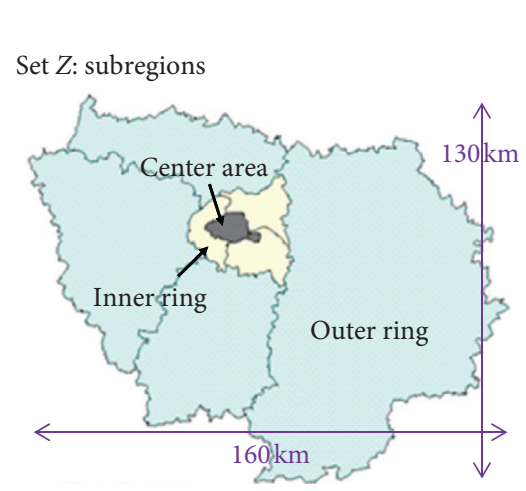

(a)
Road networks in Paris and inner suburbs

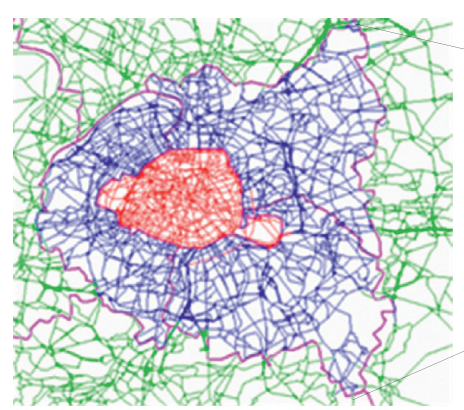

(b)

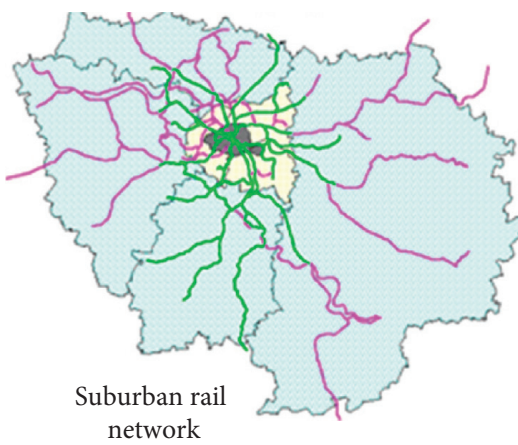

(d)

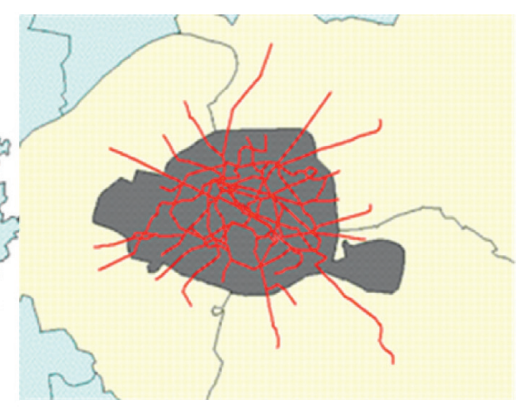

Metro network Set $R$ : transit components

(e)

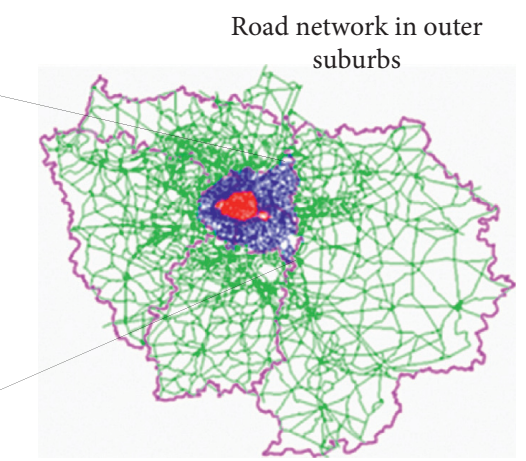

(c)

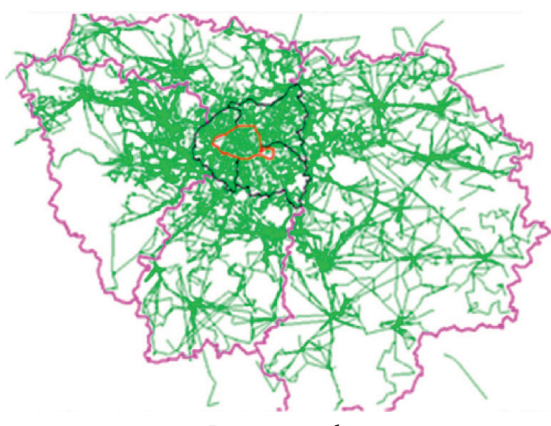

Bus network

(f)

Figure 2: Distribution into subregions, routes of the road networks, and of the transit networks.

TABLE 1: Characteristics of the road network and setting of parameters.

\begin{tabular}{lccccccc}
\hline Subarea & $l_{z}(\mathrm{~km})$ & $\Omega_{z}$ & $v_{0 z}(\mathrm{~km} / \mathrm{h})$ & $F_{z}(€)$ & Car flow M (veh/day) & VKT M (veh* $\mathrm{km} /$ day) & Current speed (km/h) \\
\hline Paris city & 1870 & 42.5 & 38 & 3.5 & 2.35 & 13.3 & 18 \\
Inner suburbs & 4417 & 54.6 & 51 & 1.0 & 6.59 & 39.2 & 30 \\
Outer suburbs & 19097 & 46.8 & 67 & 0.0 & 10.67 & 84.3 & 50 \\
\hline
\end{tabular}

TABLE 2: Featuring out transit components.

\begin{tabular}{|c|c|c|c|c|c|c|}
\hline \multirow{2}{*}{ Subarea } & \multirow{2}{*}{ Submode } & \multicolumn{3}{|c|}{ Supply factors } & \multicolumn{2}{|c|}{ Daily traffic } \\
\hline & & Network length (km) & Number of stations & Fleet size & Rides (M pax) & Distances (M pax* $\mathrm{km})$ \\
\hline \multirow{4}{*}{ Central area } & Bus & 598 & 1795 & 1295 & 1.25 & 2.58 \\
\hline & Metro & 171 & 248 & 572 & 3.97 & 15.9 \\
\hline & RER & 57 & 29 & 107 & 1.91 & 9.58 \\
\hline & Transilien & 13 & 6 & 22 & 0.62 & 1.95 \\
\hline \multirow{4}{*}{ Inner ring } & Bus & 2894 & 7575 & 3078 & 2.03 & 4.6 \\
\hline & Metro & 39 & 52 & 111 & 1.35 & 2.5 \\
\hline & RER & 181 & 85 & 129 & 2.06 & 14.3 \\
\hline & Transilien & 123 & 40 & 90 & 0.77 & 5.09 \\
\hline \multirow{3}{*}{ Outer ring } & Bus & 20032 & 25173 & 4271 & 1.24 & 4.29 \\
\hline & RER & 355 & 128 & 107 & 1.13 & 8.72 \\
\hline & Transilien & 761 & 187 & 207 & 0.65 & 8.24 \\
\hline Total & & & & & & 77.8 \\
\hline
\end{tabular}

Per year, transit production costs approximately $€ 8$ billion, whereas customer revenues bring in only $€ 2.5$ billion. The transit agency covers the operators' shortfall with an annual subsidy of $€ 5.7$ billion. For each trip, the subsidy represents an average of $€ 2$, almost twice as much as the average price. 
4.4. Environmental Impacts. By kind of vehicles, the emissions of air pollutants are derived from traffic quantities on the basis of specific emission factors, with respect to the average trip speed.

Energy consumption is modeled in relation to the traffic of cars, buses, and trains. As of 2010 in the region, while the trains had electric motors, the cars and the buses were internal combustion vehicles. The average car speeds in the different subregions entailed consumption levels of 0.10 , 0.08 , and 0.06 litre per $\mathrm{km}$ (for an average automobile type). The bus consumption factor amounts to 0.46 litre $/ \mathrm{km}$. At around $3 \mathrm{~kg} \mathrm{CO}$ per litre of fuel, the respective carbon emission factors were therefore from 0.3 down to $0.2 \mathrm{~kg}$ $\mathrm{CO}_{2} / \mathrm{km}$ for a car and $1.38 \mathrm{~kg} \mathrm{CO} / \mathrm{km}$ for a bus. As for railway vehicles, the energy consumption factors amounted to $5.9 \mathrm{kWh} / \mathrm{km}$ for a metro and $13.5 \mathrm{kWh} / \mathrm{km}$ for a suburban train. From the energy mix in France, the carbon footprint of electricity production was of $0.053 \mathrm{kgCO}_{2} / \mathrm{kWh}$.

Both air pollutant emissions and carbon emissions were monetized using the shadow price values of the French Strategic Analysis Centre [37]. The shadow prices account for emissions not only on-site but also "upstream," i.e., in the construction stages of the vehicles' lifecycles. The shadow price of air pollution involves the urban density in the emission zone, in a roughly proportional way. Table 3 gives the environmental costs of the motorized traffic depending on the vehicle kind and the impact zone, on annual basis as of 2010. The total environmental cost amounts to $€ 5.3$ billion in annual damage. Of this total, $90 \%$ is attributable to cars and $10 \%$ to buses. GHG emissions constitute a small proportion, at $6 \%$ of the total, compared with local pollution, which accounts for $94 \%$, among which 78 points are attributable to the car and 16 points to road-based transit (internal combustion buses). According to geographical zones, the centre bears $37 \%$ of the pollution, i.e., $€ 975$ per inhabitant, the inner suburbs bear $41 \%$, i.e., $€ 500$ per inhabitant, and the outer suburbs bear $12 \%$, i.e., $€ 210$ per inhabitant.

4.5. Macroeconomic Indicators. We have already measured the economics of the transit mode: per year, $€ 8$ billion in production costs, as against $€ 2.5$ bn in revenues and therefore $€ 5.7 \mathrm{bn}$ in subsidies. As for the car mode, energy expenditure amounts to $€ 3.6$ bn within a total of $€ 15$ bn of private costs. The environmental damage caused by transport costs an equivalent of $€ 5$ bn.

Still on annual basis, the generalized costs are $€ 21$ bn for transit dependent people (i.e., $€ 14$ per trip), 7.5 bn for car dependent people (i.e., $€ 1$ per trip), and $€ 27$ bn for flexible users (i.e., $€ 3.4$ per trip): in all, $€ 57 \mathrm{bn}$, of which there are 31 in the transit mode and 26 in the private car.

Thus, the global picture is dominated by the generalised costs to transport users: their magnitude is of one order greater than the costs of transit production and of environmental damage. Within the users' generalised costs, money expenditure represents one-third only, whereas the two other thirds measure the money value of the time spent by the users to travel.
The amount of users' generalised costs can be compared to the regional gross product of $€ 600 \mathrm{bn}$ in 2010: the ratio is almost $10 \%$. Furthermore, compared to the regional population, the average generalised travel cost is about $5000 €$ per person.

\section{Exploration of Mobility Policies}

The model enables us not only to represent the system in a benchmark state and to establish a diagnosis, but also to simulate the system's response to policies that act on the different instruments mentioned. In this section, we explore the potential impacts of a range of management policies, divided into three groups: first, pricing policies that target transit or the automobile mode (set 1) and then mixed policies of investment and pricing for transit, either shortterm focusing on service frequency (set 2) or long-term working in addition on the component length of lines and number of stations (set 3).

Each policy was assessed in economic terms with respect to user surplus, the profits of transit operators, the cost to the environment, and the cost to the public finances. We will present the results for each set of scenarios in succession, before undertaking an overall comparison.

\subsection{Pricing Policies}

5.1.1. Scenario 1: Free Public Transit. There is a societal debate in Île-de-France and in other cities in France, Germany, and other countries over whether to supply public transit free of charge [41]. The hoped-for benefits are an increase in the use of public transport, a reduction in environmental damage as a result of the modal shift from the car, and the reduction in the costs of fare collection. Contrarily, a rise in transit traffic would result in increased operating costs and could increase congestion or create a need for further investment. Moreover, other financial resources would have to be found to replace fare revenues, all the more so as the network is more developed.

Our first scenario is to make transit in Île-de-France free of charge, while maintaining the composition of the supply at its benchmark level. Use of transit would increase by $.3 \mathrm{M}$ trips per day, i.e., about $10 \%$ of their frequentation. Car trips would fall by the same amount: road traffic would fall down to 116 Mveh.km per day. By the resulting reduction of 5 Mveh.km per day, speeds would increase in every zone $(+2 \mathrm{~km} / \mathrm{h}$ in central area and $1^{\text {st }}$ ring, $+1 \mathrm{~km} / \mathrm{h}$ in $2^{\text {nd }}$ ring) and the pollution cost would fall by $5 \%$ ( $4 \%$ as a result of traffic reduction and $1 \%$ from better traffic conditions). The environmental gain is valued at $€ 0.22$ billion per year, which would be added to the $€ 2.8$ bn per year of additional benefits to users. However, the $€ 2.4$ bn per year of fare revenues missing from the budget would have to be financed. For the public finances, gaining $€ 0.22$ billion on the environmental side at the cost of $€ 2.4$ billion of public money is not a very viable option.

5.1.2. Scenario 2: Surtax on Fuel. We simulated a $€ 0.07$ per litre increase in the price of automobile fuel, i.e., an additional cost of 5\% on top of the TICPE (Domestic Tax on the 
TABle 3: Environmental costs of motorized modes in 2010 in Ilede-France region.

\begin{tabular}{lcccc}
\hline & \multicolumn{2}{c}{ In $€$ bn } & \multicolumn{2}{c}{ In $€ / \mathrm{p}$} \\
Subarea & Transit & Car & 2 modes & Per capita \\
\hline Central area & 0.32 & 1.63 & 1.95 & 975 \\
Inner ring & 0.17 & 2.13 & 2.3 & 500 \\
Outer ring & 0.03 & 1 & 1.03 & 210 \\
Total & 0.52 & 4.76 & 5.28 & 459 \\
\hline
\end{tabular}

Consumption of Energy Products), which French motorists feel to be high. Such a measure would have the effect of slightly reducing car traffic $(-1 \%)$ as well as total user surplus $(-1 \%)$ and environmental damage $(-1 \%)$. It would have a positive effect on the public finances, by an amount of $€ 0.35$ billion per year.

5.1.3. Scenarios 3 and 4: Urban Toll in the Central Zone. Whereas a fuel surtax would affect all car trips (since electric vehicles are very rare in the benchmark situation), an urban toll targeting the central zone would aim to reduce car traffic locally and therefore the environmental damage and inconvenience caused to residents. We used the model to find the price per unit of distance that would optimise the surplus for users and operators, in Scenario 3, or the total surplus also including the environment, in Scenario 4. The respective values would be $€ 0.26$ per kilometre and $€ 0.77$ per kilometre: including the environmental factor triples the amount compared with the scenario where only road congestion is considered. This ratio balances out the respective consequences of a modal shift of $0.5 \%$ or $1.5 \%$ of trips to public transit, toll revenues of $€ 0.49$ or 1.1 billion per year, and additional fare revenues of $€ 0.03$ or 0.09 billion per year for transit. The environmental gain would be $4.2 \%$ or $12.9 \%$, three quarters of which come from the reduction in the volume of traffic, and the remaining quarter comes from improvements in speeds that would occur locally as a result.

5.1.4. Summary of the Pricing Policies. Table 4 indicates the variations of the different scenarios from the reference state regarding operators' profits, users' surplus, environmental benefits, users' generalised costs, and specific environmental impacts. The impact on the environment varies from very small (1\% reduction in damage) for the fuel surtax, to moderate for the triple urban toll (13\%), passing by a modest level (4\%) for a single urban toll and for free transit. One idea could be to combine the instruments in order to accumulate the impacts: the risk of this would be to increase social opposition, whereas it would be fairer to ask everyone to contribute to a global objective. A road toll in the central zone with a triple level that is very high would bring in $40 \%$ of the fare revenues from transit in the benchmark situation, namely, $12 \%$ of transit production costs.

Among the benefits to users, we should recall the prime importance of the time factor in the evaluation of the generalised cost and therefore the surplus.
5.2. Short-Term Optimisation of Public Transit. Our model also enables us to simulate adaptations to the management of transit, in terms of both physical supply and fare levels, and to look for optimum management strategies. In the second set of scenarios, we considered both resizing the vehicle fleets (hence, adjusting the frequencies) and setting the fares according to the different geographical segments of demand. This resembles a value pricing strategy, but one that considers not only the production costs, but also all the commercial revenues, the modal competition with the car and the user surplus: we first optimise a target function $P^{o u}=$ $P^{u}+P^{o}$ and second the function $P^{\text {oue }}=P^{u}+P^{o}+P^{e}$ which also includes the environmental impacts. In addition, we looked at two cases, respectively, with and without the limitation of public subsidy for transit. In the case of a subsidy constraint, the operating deficit is limited to $S_{Y}=$ $€ 5.7$ billion per year, which adds a constraint of $P_{Y}^{o}+S_{Y} \geq 0$ to the simulation.

At the aggregated level (see Table 5), applying the budget constraint has a bigger impact than including the environment. Short-term optimisation would reduce the environmental cost by $4 \%$ or $6 \%$, namely, a fairly small improvement with respect to the scale defined earlier. Relaxing the budgetary constraint allows a boost to transit investment: it would be good to allocate a further $€ 3$ billion per year, which would increase transit usage by $6 \%$ (versus $1 \%$ in the constrained situation) and would improve the user surplus by $7 \%$ (versus $2 \%$ in the constrained case). The collective surplus $P^{\text {ou }}$ would grow by $1.5 \%$ or $1.8 \%$, respectively, with or without constraint, and $P^{\text {oue }}$ by $1.8 \%$ or $2.1 \%$.

Let us now set out the variations in the supply plan for the different transit components, in terms of frequency of service, which is here linked directly with fleet size. Figure 3 shows that the short-term strategies would tend

(1) in the central zone, to reduce frequencies except for suburban trains

(2) in the inner suburbs, to significantly increase bus frequency but to slightly reduce the frequency of rail modes

(3) in the outer suburbs, to increase the frequencies of all the submodes, which would therefore suggest overall improvements to transit

As for the tariff structure, the model suggests some original arrangements for the scenarios under budget constraint. To give an idea, here are the results of Scenario 6, maximisation of the total wellbeing $P^{\text {oue }}$ under the constraints of transit subsidies, nonnegativity of prices, and price cap at $€ 6$ per trip:

(1) For long trips in excess of $10 \mathrm{~km}$, null fares are found, certainly for promoting a modal shift from the car to transit modes and therefore avoiding the environmental damage caused by long car trips

(2) For short trips of less than $3 \mathrm{~km}$, a charge of $€ 2$ or $€ 3$ for every trip is imposed depending on the origin- 
TABLE 4: Assessment of $1^{\text {st }}$ scenario set: sectorial profit variations from reference state ( $€$ bn per year).

\begin{tabular}{|c|c|c|c|c|c|c|}
\hline Indicator & $\begin{array}{l}2010 \text { state } \\
\text { (references) }\end{array}$ & & $\begin{array}{l}\text { Scenario 1: free } \\
\text { transit fares }\end{array}$ & $\begin{array}{c}\text { Scenario 2: energy } \\
\text { tax }+7 \mathrm{c} € / \mathrm{L}\end{array}$ & $\begin{array}{l}\text { Scenario 3: road } \\
\text { charge for max Pou }\end{array}$ & $\begin{array}{c}\text { Scenario 4: road } \\
\text { charge for max Poue }\end{array}$ \\
\hline Transit fare revenues & 2.38 & & & 2.4 & 2.4 & 2.5 \\
\hline $\begin{array}{l}\text { Transit production } \\
\text { costs }\end{array}$ & -8.1 & & -8.2 & -8.1 & -8.1 & -8.2 \\
\hline Operator's profit Po & -5.72 & $\Delta \mathrm{Po}$ & -2.5 & 0.4 & 0.4 & 1 \\
\hline User's surplus $\mathrm{Pe}$ & -56.6 & $\Delta \mathrm{Pu}$ & 2.9 & -0.3 & -0.4 & -1.3 \\
\hline $\begin{array}{l}\text { Environmental } \\
\text { surplus Pe }\end{array}$ & -5.28 & $\Delta \mathrm{Pe}$ & 0.2 & 0.05 & 0.2 & 0.7 \\
\hline$W 1=\mathrm{Pu}+\mathrm{Po}$ & 62.3 & $\Delta$ Pou & 0.4 & 0.04 & 0.1 & -0.3 \\
\hline$W 0=\mathrm{Pu}+\mathrm{Po}+\mathrm{Pe}$ & -67.6 & $\Delta$ Poue & 0.6 & 0.09 & 0.3 & 0.4 \\
\hline TICPE revenues & & $\Delta \mathrm{ER}$ & -0.08 & 0.4 & -0.06 & -0.15 \\
\hline $\begin{array}{l}\text { Road charge } \\
\text { revenues }\end{array}$ & & $\Delta \mathrm{RCR}$ & & & 0.5 & 1.1 \\
\hline
\end{tabular}

TABLE 5: Sectorial surplus variations from reference state, in $€$ bn per year.

\begin{tabular}{|c|c|c|c|c|}
\hline & $\Delta U$ & $\Delta O$ & $\Delta E$ & $\Delta \Sigma$ \\
\hline $1 /$ free transit fares & +2.9 & -2.5 & +0.2 & +0.6 \\
\hline 2/energy tax $+7 \mathrm{c} € / \mathrm{L}$ & -0.3 & +0.37 & +0.05 & +0.1 \\
\hline $3,4 /$ road charge in central area, 0.26 OR $0.77 € / \mathrm{km}$ & $\begin{array}{l}-0.4 \\
-1.3 \\
\end{array}$ & $\begin{array}{l}+0.4 \\
+0.1 \\
\end{array}$ & $\begin{array}{r}+0.2 \\
+0.7 \\
\end{array}$ & $\begin{array}{l}+0.3 \\
+0.4 \\
\end{array}$ \\
\hline 5-8/short-run optimisation, under budget constraint OR NOT & $\begin{array}{l}1 \\
4 \\
\end{array}$ & $\begin{array}{l}0 \\
3 \\
\end{array}$ & $\begin{array}{c}+0.25 \\
+0.3\end{array}$ & $\begin{array}{l}+1.3 \\
+1.4 \\
\end{array}$ \\
\hline 9-12/long-run optimisation, under budget constraint OR NOT & $\begin{array}{l}3 \\
6 \\
\end{array}$ & $\begin{array}{c}0 \\
-2.7 \\
\end{array}$ & $\begin{array}{c}+0.25 \\
+0.3 \\
\end{array}$ & $\begin{array}{l}+3.6 \\
+3.9 \\
\end{array}$ \\
\hline
\end{tabular}

destination pair, in order to generate sufficient commercial revenues

(3) For intermediate trips over a range of between 3 and $10 \mathrm{~km}$, an intermediate charge ranging from $€ 0.5$ to $€ 2$ is imposed, except in the outer suburbs, where it would be $€ 6$ per trip (i.e., capped from above)

In other words, the model suggests reversing the intuitive logic of payment on the basis of the distances covered, in order to protect the environment while maintaining a target level of sales revenues.

In the alternative scenarios where transit subsidy is not subject to any limitation, then the optimum transit fares are close to zero: thus, these short-term scenarios encompass free fares as well as supply development for the transit mode.

5.3. Long-Term Optimisation of Transit. In the third set of scenarios, we try to optimise public transport provision by means of tariff setting together with supply development in terms of not only the size of the vehicle fleets but also the component length of lines and spacing between stations. We again compared two target functions, $P^{\text {ou }}$ or $P^{\text {oue }}$, with two cases of subsidy with or without limitation at yearly level $S_{Y}$. This results in four scenarios numbered from 9 to 12 .

The main economic results are presented in Table 5. At aggregated level, once again applying the budgetary constraint proves to be more critical than including the environment. Long-term optimisation would reduce the environmental cost to a moderate but significant degree: $9 \%$ or $12 \%$ depending on whether the subsidy is limited or not. This is twice the reduction brought by short-term optimisation. As regards the collective surpluses, long-term optimisation produces three times greater benefits than short-term optimisation, with a gain of $5 \%$ or $10 \%$. There would also be an investment boost for transit, yet somewhat smaller $(-20 \%)$ than under shortterm optimisation.

5.4. Short-Term versus Long-Term Transit Policies. In detail, depending on the components, there is a significant difference between the instruments to deploy for the long-term and those to use for the short term (see Figure 3):

(1) It would be relevant to reconfigure the bus networks by reducing the total length of lines in the three subregions and to develop all the rail submodes in all the three subregions, apart from the suburban train in the outer suburbs. This gives credence to both the recent development of tramlines in the suburbs and the Grand Paris Express project which will reinforce rail modes, especially in the inner suburbs.

(2) It would also be beneficial to reconfigure lines through a rearrangement of the stations, increasing the spacings between bus stops while reducing those between stations on rail modes.

(3) As for service frequency (neglecting capacity considerations), the model suggests that frequency should be reduced in the central zone and in the inner suburbs, except in the case of the bus (see the 


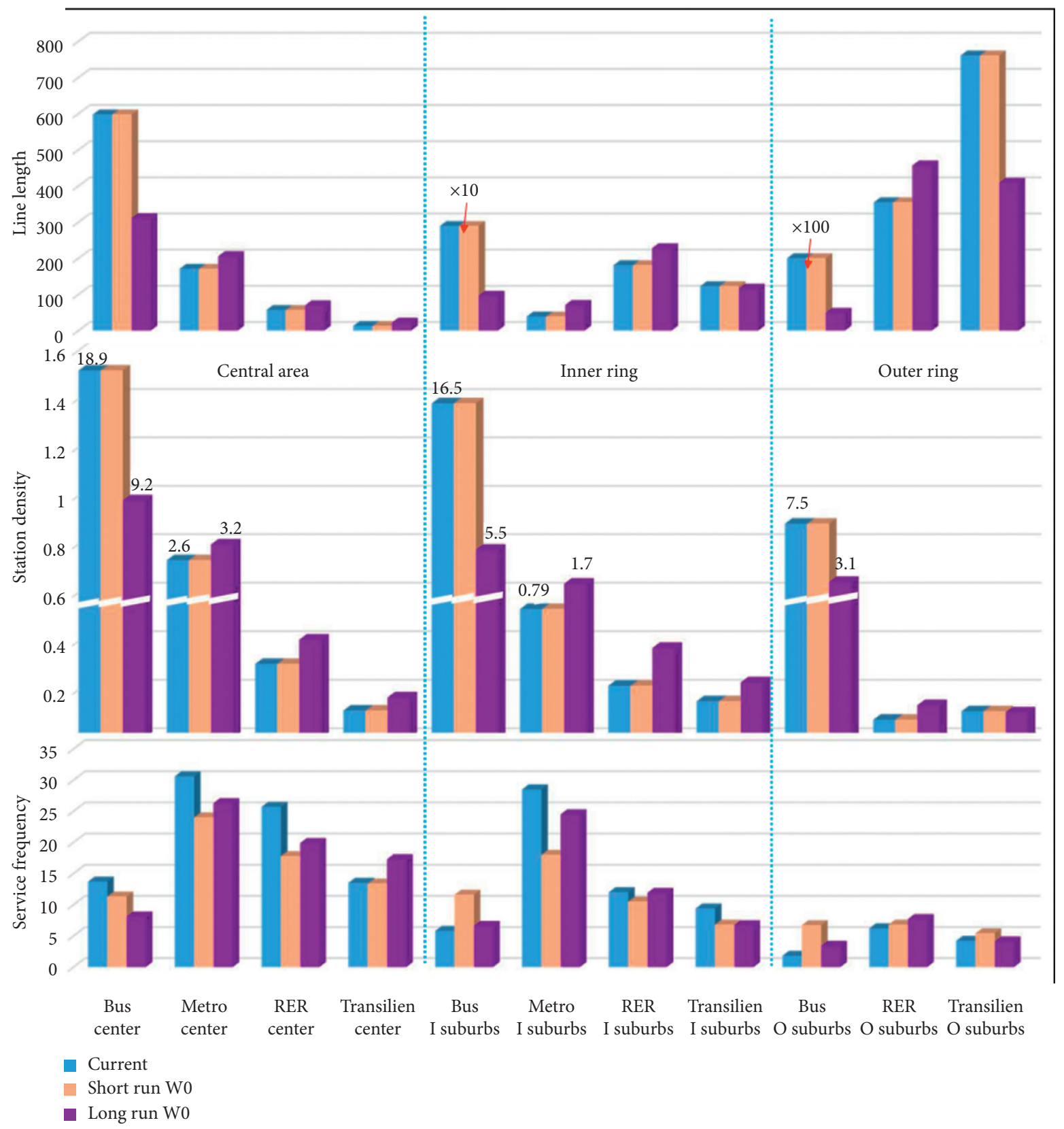

Figure 3: Transit physical supply depending on the time horizon (Scenario 6 for short term and Scenario 10 for long term).

recent development of tramlines in the Paris urbanised area), but that should be increased in the outer suburbs.

To sum up, the model suggests that it would be best to increase the provision of public rail transport in the suburbs and to reconfigure bus provision.

As for transit fares, the long-term structure in Scenario 10 (maximizing all benefits including the environmental ones under limited subsidy to transit supply) is very close to its short-term counterpart under Scenario 6. A similar correspondence holds between long-term Scenarios 9 and 11 (unconstrained) and their short-term counterparts Scenarios 5 and 7, respectively: null fares are obtained.
Figure 4 presents an analysis of costs per unit of traffic (passenger.km), for the different components and the time horizon between the benchmark state and the short term or long term (Scenarios 6 and 10, resp.). Each bar superimposes the production cost and environmental cost. Production cost depends heavily on the submode: between $€ 0.4$ and $€ 0.6$ per p.km for the bus, as compared with $€ 0.3 / \mathrm{p}$. km for the metro and between $€ 0.1$ and $€ 0.3 / \mathrm{p}$. $\mathrm{km}$ for the train. Economies of density have a significant effect in the central zone compared with the rings. Environmental costs are even more dependent on the submode, both because of the transport technology (engine type, road, or rail infrastructure) and because of the spatial configuration of the mode in terms of line length, run speed, and usage volume: between $€ 0.1$ and $€ 0.3 /$ 


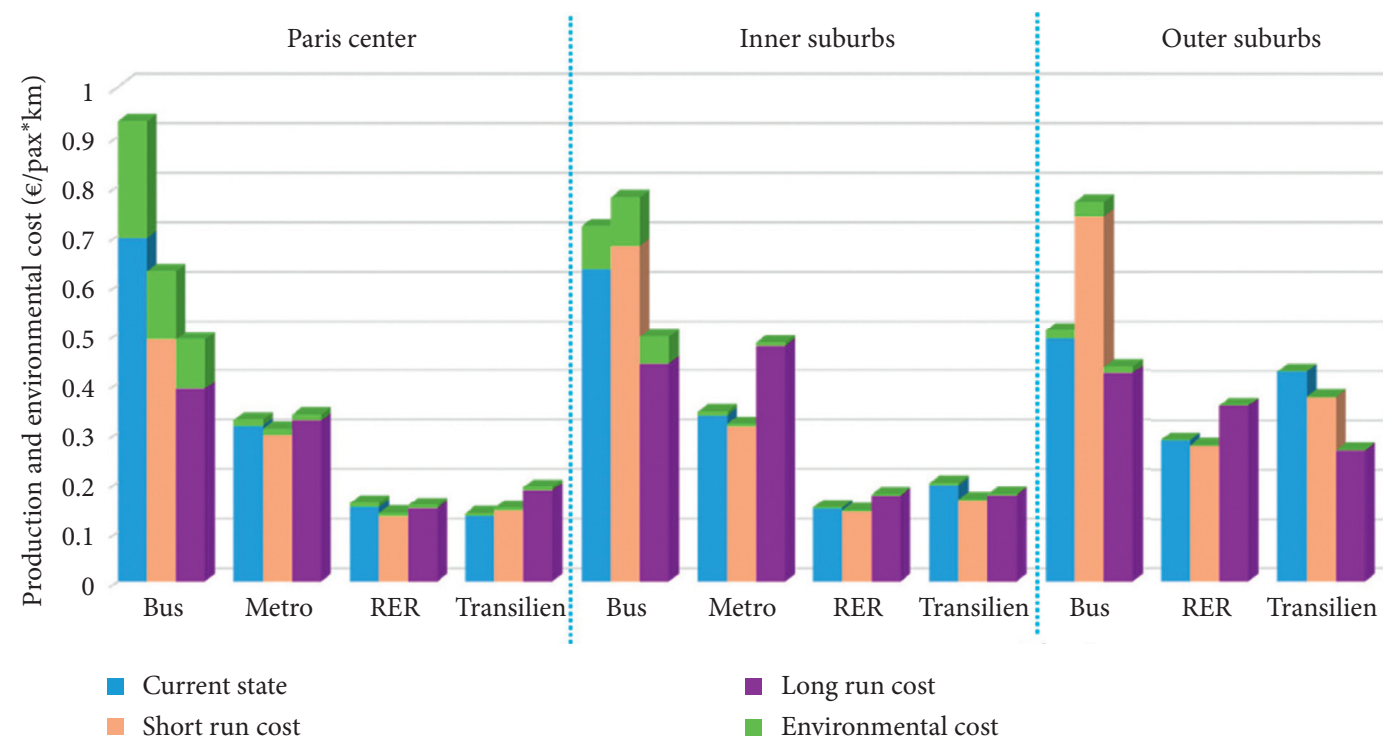

FIgURE 4: Costs to transit supply and to the environment, per passenger.km.

p.km for the bus in the central zone and in the inner ring, but for rail modes amounts of the order of a few Euro cents per p.km.

The model steers the development of transit supply towards a significant reduction in the environmental costs for bus trips, by reconfiguring provision between transit components in addition to fostering a modal shift from the car to transit. It should be recalled that the environmental cost of the automobile mode is $€ 0.5$ per vehicle. $\mathrm{km}$ in the central zone or $€ 0.2$ per vehicle.km in the suburbs, i.e., markedly higher per passenger than by the bus and two orders of magnitude higher than by rail modes.

\section{Conclusion}

The study contribution is threefold, from strategic model to global picture of regional mobility system and up to prospective hints for mobility policies.

The strategic model constitutes a simplified analytical framework to understand the composition of the motorized mobility system in a regional territory and to make an economic assessment of its stakes. The current version encompasses the private car and transit modes and the users' choices between them: these are the currently dominant modes for medium to long range trips and their share in passenger traffic is vastly dominant. The demand model involves trip segmentation both geographical and behavioral but no activity purposes nor activity and trip chaining. The model composition allows for consideration of the two pricing principles of "user pays" and "polluter pays": further development will involve the identification of firms as benefiters of commuting trips and business purposes, beyond their employees.

Coming to the global picture of motorized mobility in the Paris Ile-de-France region, the strategic model eases the identification of relevant macroeconomic indicators. The balance sheet is dominated by the generalized cost of trips to theirs users and among these the valuation of travel times. Then, by decreasing order of magnitude, come the money costs of private cars to their users, the transit production costs to its operators, the public subsidies to the transit mode, the environmental costs, energy costs to car users, and the commercial revenues of transit fares. The amount of transit subsidies almost matches the environmental costs that come primarily from car traffic: this unexpected outcome suggests some balance of public policy between the two motorized costs: in both cases, the community pays for the costs in the place of their users. The question of fairness between mobility users and tax-payers requires further modelling and assessment. Concerning environmental damage, we showed that there is a 5:1 ratio of experienced cost per capita between central area dwellers and outer suburbanites, quite a large spatial inequality.

The global picture is strongly dependent on the shadow prices associated with travel time values, on the one hand, and with environmental damage, on the other hand. The recent restatement of carbon prices for France at the 2030 time horizon [42] would make carbon emission rise from 6\% to one-third of environmental costs, which would increase by one quarter. The resulting total would still be much lower than users' time costs, thereby raising the question of reevaluating the values of time and bringing them closer to real-world willingness-to-pay. Further research is required for revealing behavioral evidence in this respect.

Turning to the prospective study of mobility policy making, road charging at high price would be the most effective instrument to combat the environmental damage of car traffic, if acceptable. Yet, this would curb only $15 \%$ of environmental costs. Making the transit mode free of charge to its users would achieve half of this abatement only, while having a much higher cost to the public purse. Further development is required in global system optimisation in order to endogenize the limited availability of public funds. 
Application of the model in the current version also suggests developing the transit network by a significant extent (10\% more spending) in both the medium and long run. Further research is required to consider policy packages combining road pricing and transit development and also to consider the electrification of vehicle fleets, both for buses and for private cars.

\section{Data Availability}

There are three datasets underpinning the study: first, the Household Travel Survey "EGT 2010" which belongs to IDFM, the Ile-de-France Mobility Organizing Authority; second, the network databases of roadways and transit services in the MODUS model, which belongs to DRIEA; and, third, accountancy statistics about transit networks in the Ile-de-France region. None of these datasets are available on a public basis.

\section{Additional Points}

Highlights. Strategic model of mobility system including car and public transit modes. System optimization considering demand surplus, supply profit, and environmental costs. Assessment and hierarchy of mobility stakes in the Paris region as of 2010. Exploration of short-, medium-, and longterm policies.

\section{Disclosure}

This research was performed as part of the employment of the authors at Ecole des Ponts ParisTech.

\section{Conflicts of Interest}

The authors declare that there are no conflicts of interest regarding the publication of this article.

\section{Authors' Contributions}

Both authors are fully and only responsible for manuscript writing, editing, approval, and submittal for publication.

\section{Acknowledgments}

The authors express their gratitude to both Supporting Chairs: IDFM for making the Household Travel Survey (EGT) available and DRIEA for providing the "MODUS" travel demand model. The employment frame of Sheng Li was supported by two Research Chairs at École des Ponts ParisTech: the Chair in the Eco-design of Built Sets and Infrastructures, sponsored by the VINCI Group, and the Chair in Territorial Mobility, sponsored by Île-de-France Mobilités (IDFM).

\section{References}

[1] Rupprecht Consult, Ed., Guidelines for Developing and Implementing a Sustainable Urban Mobility Planp. 166, 2019, https://www.eltis.org/sites/default/files/sump-guidelines-2019_ mediumres.pdf, 2nd edition.
[2] F. Leurent, S. Li, and H. Badia, "Structural design of a hierarchical urban transit network integrating modal choice and environmental impacts," Transportation Research Procedia, vol. 37, pp. 99-106, 2019.

[3] J. Dupuit, "De la mesure de l'utilité des travaux publics," Annales des Ponts et Chaussées, 2e série, Mémoires et documents, vol. 8, no. 116, pp. 332-375, 1844.

[4] A. C. Pigou, The Economics of Welfare, Macmillan, London, UK, 1st edition, 1920.

[5] J. G. Wardrop, "Road paper. Some theoretical aspects of road traffic research," Proceedings of the Institution of Civil Engineers, vol. 1, no. 3, pp. 325-362, 1952.

[6] A. A. Walters, "The theory and measurement of private and social cost of highway congestion," Econometrica, vol. 29, no. 4, pp. 676-699, 1961.

[7] W. S. Vickrey, "Congestion theory and transport investment," The American Economic Review, vol. 59, no. 2, pp. 251-260, 1969.

[8] H. Mohring, "Optimization and scale economies in urban bus transportation," The American Economics Review, vol. 62, no. 4, pp. 591-604, 1972.

[9] M. J. Beckmann, C. McGuire, and C. Winsten, Studies in the Economics of Transportation, Yale University Press, London, UK, 1956.

[10] H. Spiess and M. Florian, "Optimal strategies: a new assignment model for transit networks," Transportation Research Part B: Methodological, vol. 23, no. 2, pp. 83-102, 1989.

[11] M. Cepeda, R. Cominetti, and M. Florian, "A frequency-based assignment model for congested transit networks with strict capacity constraints: characterization and computation of equilibria," Transportation Research Part B: Methodological, vol. 40, no. 6, pp. 437-459, 2006.

[12] Z.-C. Li, H.-J. Huang, W. H. K. Lam, and S. C. Wong, "A model for evaluation of transport policies in multimodal networks with road and parking capacity constraints," Journal of Mathematical Modelling and Algorithms, vol. 6, no. 2, pp. 239-257, 2007.

[13] M. Kuwahara and T. Akamatsu, "Decomposition of the reactive dynamic assignments with queues for a many-to-many origin-destination pattern," Transportation Research Part B: Methodological, vol. 31, no. 1, pp. 1-10, 1997.

[14] L. Meschini, G. Gentile, and N. Papola, "A frequency based transit model for dynamic traffic assignment to multimodal networks," in Proceedings of the 17th International Symposium on Transportation and Traffic Theory (ISTTT), M. G. H. Bell, B. G. Heydecker, and R. E. Allsop, Eds., Elsevier, London, UK, pp. 407-436, July 2007.

[15] J. d. D. Ortùzar and L. G. Willumsen, Modelling Transport, John Wiley \& Sons, Hoboken, NJ, USA, 4 edition, 2011.

[16] L. J. LeBlanc, "An algorithm for the discrete network design problem," Transportation Science, vol. 9, no. 3, pp. 183-199, 1975.

[17] G. E. Cantarella and A. Vitetta, "The multi-criteria road network design problem in an urban area," Transportation, vol. 33, no. 6, pp. 567-588, 2006.

[18] Y.-J. Lee and V. R. Vuchic, "Transit network design with variable demand," Journal of Transportation Engineering, vol. 131, no. 1, pp. 1-10, 2005.

[19] A. T. Murray, "A coverage model for improving public transit system accessibility and expanding access," Annals of Operations Research, vol. 123, no. 1/4, pp. 143-156, 2003.

[20] R. Van Nes, "Design of multimodal transport networks a hierarchical approach,” T2002/5, Trail Thesis Series, Delft University Press, Delft, Netherlands, 2002. 
[21] F. Combes and R. van Nes, "A simple representation of a complex urban transport system based on the analysis of transport demand: the case of region Ile-de-France," Procedia-Social and Behavioral Sciences, vol. 48, pp. 3030-3039, 2012.

[22] F. Leurent, F. Combes, and R. van Nes, "From strategic modelling of urban transit systems to golden rules for their design and management," 2016, https://hal.archives-ouvertes. $\mathrm{fr} / \mathrm{hal}-02462463$.

[23] C. F. Daganzo, "Structure of competitive transit networks," Transportation Research Part B: Methodological, vol. 44, no. 4, pp. 434-446, 2010.

[24] M. Estrada, M. Roca-Riu, H. Badia, F. Robusté, and C. F. Daganzo, "Design and implementation of efficient transit networks: procedure, case study and validity test," Transportation Research Part A: Policy and Practice, vol. 45, no. 9, pp. 935-950, 2011.

[25] H. Badia, M. Estrada, and F. Robusté, "Competitive transit network design in cities with radial street patterns," Transportation Research Part B: Methodological, vol. 59, pp. 161181, 2014.

[26] H. Badia, M. Estrada, and F. Robusté, "Bus network structure and mobility pattern: a monocentric analytical approach on a grid street layout," Transportation Research Part B: Methodological, vol. 93, pp. 37-56, 2016.

[27] W. Zhang, E. Jenelius, and H. Badia, "Efficiency of semiautonomous and fully autonomous bus services in trunk-andbranches networks," Journal of Advanced Transportation, vol. 2019, p. 17, Article ID 7648735, 2019.

[28] C. F. Daganzo and N. Geroliminis, "An analytical approximation for the macroscopic fundamental diagram of urban traffic," Transportation Research Part B: Methodological, vol. 42, no. 9, pp. 771-781, 2008.

[29] N. Geroliminis and C. F. Daganzo, "Existence of urban-scale macroscopic fundamental diagrams: some experimental findings," Transportation Research Part B: Methodological, vol. 42, no. 9, pp. 759-770, 2008.

[30] N. Geroliminis and J. Sun, "Properties of a well-defined macroscopic fundamental diagram for urban traffic," Transportation Research Part B: Methodological, vol. 45, no. 3, pp. 605-617, 2011.

[31] V. L. Knoop, H. Van Lint, and S. P. Hoogendoorn, "Traffic dynamics: its impact on the macroscopic fundamental diagram," Physica A: Statistical Mechanics and Its Applications, vol. 438, pp. 236-250, 2015.

[32] J. A. Laval and F. Castrillón, "Stochastic approximations for the macroscopic fundamental diagram of urban networks," Transportation Research Part B: Methodological, vol. 81, pp. 904-916, 2015.

[33] N. Zheng, R. A. Waraich, K. W. Axhausen, and N. Geroliminis, "A dynamic cordon pricing scheme combining the macroscopic fundamental diagram and an agentbased traffic model," Transportation Research Part A: Policy and Practice, vol. 46, no. 8, pp. 1291-1303, 2012.

[34] B. Wei and D. Sun, "A two-layer network dynamic congestion pricing based on macroscopic fundamental diagram," Journal of Advanced Transportation, vol. 2018, p. 11, Article ID 8616120, 2018.

[35] M. Estrada, F. Robusté, J. Amat, H. Badia, and J. Barceló, "Optimal length of transit network with traffic performance microsimulation," Transportation Research Record: Journal of the Transportation Research Board, vol. 2276, no. 1, pp. 9-16, 2012.
[36] S. Nguyen and S. Pallottino, "Equilibrium traffic assignment for large scale transit networks," European Journal of Operational Research, vol. 37, no. 2, pp. 176-186, 1988.

[37] E. Quinet et al., "L'évaluation socioéconomique des investissements publics," Rapport du Commissariat général à la stratégie et à la perspective, République Française, France, Europe, 2013.

[38] F. Leurent and S. Li, "Un modèle stratégique de mobilité: application à l'Ile de France. Communication au congrès RDMI 2019," 2019.

[39] Copert, EMEP/EEA Air Pollutant Emission Inventory Guidebook, 2016, https://www.eea.europa.eu/publications/ emep-eea-guidebook-2016.

[40] Omnil, "Enquête globale transport: la mobilité en Ile de France," 2012.

[41] J. Rapoport et al., "Rapport du Comité sur la faisabilité de la gratuité des transports en commun en Île-de-France, leur financement et la politique de tarification," 2018.

[42] A. Quinet et al., "La valeur de l'action pour le climat," Rapport du Commissariat général à la stratégie et à la perspective, République Française, France, Europe, 2019. 